\title{
Análise de vizinhança na avaliação de genótipos de cana-de-açúcar
}

\author{
Liliam Silvia Candido(1), Dilermando Perecin(2), Marcos Guimarães de Andrade Landell(3) e Bruno Ettore Pavan ${ }^{(2)}$ \\ (1)Universidade Estadual Norte Fluminense Darcy Ribeiro, Laboratório de Melhoramento Genético Vegetal, Avenida Alberto Lamego, 2.000, \\ CEP 28013-602 Campos dos Goytacazes, RJ. E-mail: Icandido@uenf.br (2)Universidade Estadual Paulista, Faculdade de Ciências Agrárias \\ e Veterinárias, Departamento de Ciências Exatas, Via de Acesso Prof. Paulo Donato Castellane s/no, CEP $14884-900$ Jaboticabal, SP. \\ E-mail: perecin@fcav.unesp.br, brunoe.pavan@ig.com.br ${ }^{(3)}$ Instituto Agronômico, Centro de Cana, Anel Viário Contorno Sul, Km 321, \\ Caixa postal 206, CEP 14001-970 Ribeirão Preto, SP. E-mail: mlandell@iac.sp.gov.br
}

Resumo - O objetivo deste trabalho foi estudar diferentes covariáveis de competição em análises de vizinhança, e verificar sua eficiência no aumento da precisão experimental e as consequências no ordenamento de genótipos de cana-de-açúcar em termos de potencial produtivo. Foram utilizados dados da rede de ensaios do programa de melhoramento de cana-de-açúcar do Instituto Agronômico, instalados em 2002. O delineamento foi em blocos ao acaso, com três repetições. As parcelas constituíram-se de cinco sulcos de $8 \mathrm{~m}$, espaçados $1,50 \mathrm{~m}$ entre si. A produtividade de colmos por hectare $\left(\mathrm{TCH}, \mathrm{Mg} \mathrm{ha}^{-1}\right)$ foi avaliada no primeiro e terceiro cortes. As covariáveis "lateral", "ponta" e "quatro vizinhos" foram incluídas nos métodos de Papadakis, em duas variações do método médias móveis. Os métodos de Papadakis e médias móveis 1 não alteraram a precisão experimental em comparação à análise tradicional. O método médias móveis 2 reduziu a variância ambiental, mas alterou as estimativas da variância genotípica e o ordenamento dos genótipos, o que pode induzir a conclusões equivocadas na seleção. Embora a covariável "lateral" tenha sido influente na competição, o efeito em parcelas de cinco sulcos é pequeno.

Termos para indexação: Saccharum, covariância, médias móveis, Papadakis, precisão experimental.

\section{Neighborhood analysis in evaluation of sugarcane genotypes}

\begin{abstract}
The objective of this work was to study different competition covariates in neighborhood analysis and to check the efficiency of this analysis in the increase of the experimental precision and the consequences in the ranking of sugarcane genotypes in terms of productive potential. Data used were from the state experimental network of the sugarcane breeding program installed in 2002, of the Instituto Agronômico, Campinas, SP, Brazil. The experimental design was in randomized complete blocks, with three replicates. The plots were formed of five $8 \mathrm{~m}$ rows with $1.5-\mathrm{m}$ spacing between rows. The stalk productivity $\left(\mathrm{TCH}, \mathrm{Mg} \mathrm{ha}^{-1}\right)$ was evaluated in the first and third harvests. The covariates "side", "tip" and "4 neighbors" were included in the Papadakis method and in two variations of the moving average method. The Papadakis and moving average 1 methods did not change the experimental precision in comparison with traditional analysis. The moving average 2 method reduced the environmental variance. However, it changed the estimates of the genetic variance and the genotype ranking, and could lead to erroneous conclusions in the selection. Although the "side" covariate was influential on competition, its effect or on five row-plots was small.
\end{abstract}

Index terms: Saccharum, covariance, moving averages, Papadakis, experimental precision.

\section{Introdução}

O sucesso dos programas de melhoramento de plantas depende, entre outros fatores, da realização de ensaios de campo e de metodologias de análise eficientes, para a correta detecção dos genótipos superiores que poderão ser lançados comercialmente.

Desde o planejamento dos experimentos, é necessário adotar medidas para minimizar os efeitos do erro experimental. Entre essas medidas estão a repetição, a casualização e o controle local dos tratamentos em teste, chamados de princípios básicos da experimentação (Storck et al., 2006). Entretanto, mesmo com esses cuidados pode ocorrer aumento indesejável do erro experimental, em razão das variações causadas por fatores não controláveis, como por exemplo, a heterogeneidade do solo ou das parcelas ou, ainda, a competição entre e dentro das parcelas experimentais (Ramalho et al., 2005).

A análise tradicional dos experimentos de campo não considera que as observações tomadas em plantas ou parcelas vizinhas sejam correlacionadas, uma vez que 
uma das pressuposições do modelo é a independência entre os erros. Assim, estratégias de análise que modelam a dependência espacial, na forma de análise de covariância, têm sido sugeridas no melhoramento de plantas, a fim de se exercer maior controle local e aumentar a precisão experimental. Nessas análises, considera-se que os efeitos do erro experimental sejam tão mais semelhantes quanto mais próximas estejam localizadas as parcelas (Costa et al., 2005). De acordo com Resende (2000), esse tipo de análise permite corrigir os dados de tal forma que as variações devidas à covariável sejam minimizadas, ou até mesmo anuladas, e que forneçam estimativas corretas dos componentes de variância e de outros parâmetros obtidos.

Os métodos de Papadakis e médias móveis têm sido os mais utilizados em dados simulados (Vivaldi, 1990) ou em dados de campo de culturas de importância agronômica, como milho, feijão e soja (Amaro et al., 1997; Souza et al., 2000; Cargnelutti Filho et al., 2003; Costa et al., 2005; Storck et al., 2008, 2009). A diferença entre os dois é que o método de Papadakis corrige o valor observado em cada parcela, pelo efeito residual existente nas parcelas vizinhas, enquanto no método das médias móveis, o valor de cada parcela é corrigido pela média dos valores das parcelas vizinhas. O número de vizinhos considerados no cálculo das covariáveis de competição também é questionável. De acordo com Vivaldi (1990), esse número é aquele até onde o processo de criação da covariável se mostra eficiente, e isto pode variar de local para local, ou mesmo dentro de um experimento.

Analisando-se o princípio da casualização, poderse-ia considerar que num experimento delineado, a análise de covariância pudesse ser ignorada. No entanto, Souza et al. (2000), Cargnelutti Filho et al. (2003) e Storck et al. (2008) evidenciaram a contribuição desse tipo de análise na redução do erro experimental e no aumento da eficiência na seleção, em diferentes culturas.

O objetivo deste trabalho foi estudar diferentes formas de cálculo de covariáveis de competição e aplicá-las nas metodologias de análise de vizinhança: Papadakis, médias móveis 1 e médias móveis 2, para verificar a eficiência dessas análises no aumento da precisão experimental, bem como as consequências no ordenamento de genótipos de cana-de-açúcar em termos de potencial produtivos, em experimentos de melhoramento genético.

\section{Material e Métodos}

Para a realização das análises, foram utilizados dados dos experimentos que constituíram a rede de ensaios do ano de 2002, conduzidos pelo programa de melhoramento de cana-de-açúcar, do Instituto Agronômico (IAC), Campinas, SP.

Os experimentos foram instalados no período de $1 / 3$ a $8 / 4 / 2002$, com a primeira colheita realizada após um ano e meio, e as demais ano a ano, em sete locais, sendo cinco municípios no Estado de São Paulo (Catanduva, Cosmópolis, Sales Oliveira, Motuca e Pradópolis), um município no Estado de Minas Gerais (Delta) e um município no Estado de Goiás (Goianésia).

Cada experimento foi instalado com 26 genótipos: 15 clones IAC (numerados de 1 a 15) e 11 cultivares padrões - três fixas (numeradas de 16 a 18), quatro móveis, que variaram de acordo com o local do experimento (numeradas de 19 a 22), e outras quatro móveis que foram avaliadas em apenas uma localidade e, por essa razão, mesmo fazendo parte das análises, foram excluídas do ordenamento.

O delineamento, em todos os experimentos, foi o de blocos completos ao acaso, com três repetições, com uso de bordaduras nas laterais dos blocos. A casualização dos genótipos em cada bloco foi feita uma única vez e repetida em todos os locais. Assim, em cada bloco, os genótipos tiveram os mesmos vizinhos em todos os locais (exceto no caso de cultivares padrões móveis). A adubação foi realizada de acordo com a recomendação de cada localidade, variado de 500 a $600 \mathrm{~kg} \mathrm{ha}^{-1}$ da fórmula 05-20-20, no plantio, e de 20-05-20 nas socas.

As parcelas experimentais foram constituídas por cinco sulcos de $8 \mathrm{~m}$, espaçadas $1,50 \mathrm{~m}$ entre si, com área útil de $60 \mathrm{~m}^{2}$. $\mathrm{O}$ atributo produtividade de colmos por hectare ( $\mathrm{TCH}, \mathrm{Mg} \mathrm{ha}^{-1}$ ) foi quantificado, tendo-se pesado todos os colmos das parcelas, sem o ponteiro, e extrapolado para representar a produção por hectare. As avaliações desse atributo foram realizadas no primeiro e no terceiro cortes, na época média de colheita entre os meses de junho e agosto (inverno). Em todos os locais, os cortes foram realizados de forma manual na cana queimada, exceto no Município de Sales Oliveira, onde a colheita foi mecânica e de cana crua.

Para fins de comparação com a análise clássica, sem a inclusão de covariáveis de competição, foram

Pesq. agropec. bras., Brasília, v.44, n.10, p.1304-1311, out. 2009 
considerados os métodos de análise de vizinhança baseados em análise de covariância, pelas metodologias Papadakis, médias móveis 1 e médias móveis 2 .

O método de Papadakis consiste em corrigir a produtividade de cada parcela pela média dos resíduos das parcelas vizinhas. Dessa forma, o resíduo de cada parcela foi calculado pela expressão: $R_{(\mathrm{i}, \mathrm{j})}=\mathrm{Y}_{(\mathrm{i}, \mathrm{j})}-\hat{\mathrm{Y}}_{(\mathrm{i}, \mathrm{,})}$, em que: $\mathrm{R}_{(\mathrm{i}, \mathrm{j})}$ corresponde ao resíduo da parcela com o i-ésimo tratamento e a j-ésima repetição; $\mathrm{Y}_{(\mathrm{i}, \mathrm{j})}$ é o valor observado de TCH na parcela com o i-ésimo tratamento e a j-ésima repetição; $\hat{\mathrm{Y}}_{(\mathrm{i}, \text {, }}$ é a média das parcelas com o i-ésimo tratamento. Os valores obtidos dos $\mathrm{R}_{(\mathrm{i}, \mathrm{j})}$ de cada parcela são posicionados segundo a casualização realizada para cada bloco, em cada um dos sete experimentos. Assim, as médias calculadas podem servir como covariável de competição.

O método das médias móveis foi utilizado segundo duas formas de cálculo de correção das parcelas, denominado, neste trabalho, de médias móveis $1 \mathrm{e}$ médias móveis 2 . O método médias móveis 1 consistiu em corrigir a produtividade de cada parcela pela média dos valores de TCH observados nas parcelas vizinhas; e o próprio valor obtido dessa média foi utilizado como covariável. No método médias móveis 2, a covariável correspondeu à diferença entre o valor de TCH observado na parcela e a média de TCH dos vizinhos. Por exemplo, considerando-se dois vizinhos, à direita e à esquerda de uma parcela qualquer, temos: $Z_{a b}=Y_{a b}-1 / 2\left(Y_{e b}+Y_{d b}\right)$, em que: $Y_{e b}$ e $Y_{\mathrm{db}}$ são valores observados à esquerda e à direita da parcela $a b$, respectivamente. Assim, $Z_{a b}$ corresponde ao valor de uma covariável $\mathrm{Z}$ associada à parcela $\mathrm{ab}$, na forma descrita por Vivaldi (1990).

Nos três modelos de análise de vizinhança descritos, foram incluídas três diferentes covariáveis de competição, isoladas e em associação. A diferença entre as covariáveis foi basicamente o número de vizinhos considerados para o cálculo da média ou resíduo médio (método de Papadakis) e a posição desses vizinhos em relação à parcela. Assim, foram obtidas as seguintes covariáveis de competição: covariável "lateral" - média ou resíduo médio de $\mathrm{TCH}$ das duas parcelas laterais à parcela objeto (vizinhos localizados à direita e à esquerda da parcela); covariável "ponta" - média ou resíduo médio de $\mathrm{TCH}$ das duas parcelas vizinhas nas pontas dos sulcos, em relação à parcela objeto (nesse caso os vizinhos podiam pertencer ou não ao mesmo bloco da parcela objeto); covariável "quatro vizinhos" - média ou resíduo médio de $\mathrm{TCH}$ das quatro parcelas mais próximas à parcela objeto. Para as parcelas localizadas nas extremidades dos blocos, a bordadura existente não foi incluída no cálculo das covariáveis e, nesses casos, foram considerados a média ou resíduo médio de uma, duas ou três parcelas vizinhas, conforme o caso.

A análise conjunta seguiu o processo de estimação-predição dos modelos mistos (Henderson, 1975), tendo-se inicialmente combinado os dados dos sete locais sem a inclusão de covariáveis de competição e, posteriormente, considerado cada covariável isoladamente e as três associadas nos métodos de vizinhança: Papadakis, médias moveis 1 e médias móveis 2 . Esse modelo é representado matricialmente por $\mathrm{y}=\mathrm{X} \beta+\mathrm{Za}+\mathrm{e}$, em que: y é o vetor $(n \times 1)$ de observações; $X$ é a matriz $(n \times p)$ de incidência dos efeitos fixos $\beta$; $Z$ é a matriz $(n \times q)$ de incidência dos efeitos aleatórios a (genéticos); $\beta$ é o vetor $(\mathrm{p} \times 1)$ de efeitos fixos a serem estimados; a é o vetor $(\mathrm{q} \times 1)$ de efeitos aleatórios ou valores genotípicos a serem preditos; e "e" é o vetor (nx1) de erros aleatórios associados a cada observação. Admitiu-se que: e $\sim \mathrm{N}_{\mathrm{n}}(0, \mathrm{R})$, em que $\mathrm{R}$ é a matriz de variâncias e covariâncias residuais, dada por $\mathrm{R}=$ I $\sigma_{\text {e }}$ e a $\sim \mathrm{N}_{\mathrm{q}}(0, \mathrm{G})$, em que $\mathrm{G}$ é a matriz de variâncias e covariâncias genotípicas, dada por: $\mathrm{G}=\mathrm{A} \sigma_{\mathrm{g}}{ }_{\mathrm{g}}$, em que: $\sigma_{\mathrm{g}}^{2}$ é a variância genotípica, e $\mathrm{A}$ corresponde à matriz de parentesco genético entre genótipos. Mas, como nos experimentos em estudo não se conhecia a genealogia dos genótipos, admitiu-se que A é igual à matriz identidade (I) e, portanto: $G=I \sigma_{g}^{2}$.

Essametodologia consistenaobtenção depredições dos valores genotípicos tratados como efeito aleatório, corrigidos para os demais efeitos fixos contidos no modelo: blocos e covariáveis de competição. As estimativas dos componentes de variância foram obtidas pelo método da máxima verossimilhança restrita (REML) descrito por Patterson \& Thompson (1971). O melhor preditor linear não viesado (BLUP) dos valores genotípicos de cada indivíduo foi obtido com uso do SAS (SAS Institute, 2002). Para a análise conjunta, o procedimento utilizado foi: Proc mixed method = REML; class local bloco genótipo; model TCH = local bloco (local) cováriavel; random genótipo genótipo*local/solution; run. 
A comparação entre os métodos de análise de vizinhança e sua eficiência em aumentar a precisão experimental, em relação à análise clássica, foi realizada com base nas estimativas da variância genotípica, variância da interação genótipos x locais, variância residual (quadrado médio do resíduo), herdabilidade no sentido amplo e coeficiente de variação. Também foram estimados os valores de significância pelo teste $\mathrm{F}$, para as três covariáveis de competição adotadas, isoladamente e associadas. $\mathrm{O}$ teste $\mathrm{F}$ equivale ao teste do coeficiente de regressão linear (b) entre a variável TCH de cada tratamento e a covariável em questão. Para os nove ordenamentos obtidos, conforme cada método de vizinhança e covariável, foram calculadas as correlações de Spearman (r) entre a ordenação de cada análise e a da análise clássica, a fim de se avaliarem as eventuais alterações de posicionamento dos genótipos nos ordenamentos.

\section{Resultados e Discussão}

Nas análises de primeiro corte (Tabela 1), os valores de variância ambiental estimada pelos métodos de Papadakis e médias móveis 1, com inclusão em separado das covariáveis "lateral", "ponta" e "quatro vizinhos", foram similares ou ligeiramente superiores (até $0,52 \%$ ) aos da variância ambiental estimada pela análise sem a inclusão das covariáveis de competição. No terceiro corte (Tabela 2), a maioria das covariáveis provocou redução na variância ambiental. No entanto, a maior redução foi inferior a $2 \%$, observada com o uso da covariável "ponta" na metodologia de Papadakis. O ligeiro aumento da variância ambiental, observado em alguns casos, normalmente não ocorre em análises de covariância. Contudo, ele pode ser explicado pelo fato de os graus de liberdade do resíduo das análises com covariáveis serem uma unidade inferior aos da análise clássica e pelo fato de os efeitos dessas covariáveis terem sido baixos nesses casos.

Costa et al. (2005) obtiveram reduções de $5,5 \%$ e $6,2 \%$, em média, na variância ambiental, respectivamente para os métodos de Papadakis e médias móveis, em experimentos com progênies de feijão e milho. No entanto, Cargnelutti Filho et al. (2003) utilizaram o método de Papadakis em ensaios de competição de cultivares de milho, e observaram reduções na variância ambiental de até $57,4 \%$, com a inclusão de cinco diferentes tipos de covariáveis no modelo. Storck et al. (2008) também relataram eficiência satisfatória do método de Papadakis na melhoria das estatísticas que retratam a precisão experimental em ensaios com soja.

Ao contrário do observado nas outras metodologias, o método médias móveis 2 com a inclusão das covariáveis provocou fortes reduções (30,4$63,9 \%$ ) na variância ambiental no primeiro corte (Tabela 1) e no terceiro-corte (31,9-56,3\%, Tabela 2). Os coeficientes de variação estimados

Tabela 1. Estimativas das variâncias genotípicas $\left(\sigma_{\mathrm{G}}^{2}\right)$, de interação genótipos x locais $\left(\sigma_{\mathrm{GxL}}^{2}\right)$ e ambiental $\left(\sigma_{\mathrm{e}}^{2}\right)$, da herdabilidade $\left(\mathrm{h}^{2}\right)$ e do coeficiente de variação $(\mathrm{CV})$, sem e com o uso de covariáveis de competição pelos métodos de Papadakis, médias móveis 1 e médias móveis 2, e valor do teste $\mathrm{F}$ para cada coeficiente de regressão (b), entre as respostas das parcelas e os valores das covariáveis de competição, para o atributo produtividade ( $\left.\mathrm{TCH}, \mathrm{Mg} \mathrm{ha}^{-1}\right)$ no primeiro corte, de 26 genótipos de cana-de-açúcar, em sete locais.

\begin{tabular}{|c|c|c|c|c|}
\hline \multirow[t]{2}{*}{ Parâmetro } & \multirow{2}{*}{$\begin{array}{c}\text { Análise sem } \\
\text { covariável }\end{array}$} & \multicolumn{3}{|c|}{ Métodos de vizinhança } \\
\hline & & Papadakis & Médias móveis 1 & Médias móveis 2 \\
\hline & & \multicolumn{3}{|c|}{ Covariável "lateral" } \\
\hline$\sigma_{\mathrm{G}}^{2}$ & 91,87 & 92,25 & 91,98 & 30,25 \\
\hline$\sigma_{\mathrm{GxL}}^{2}$ & 38,14 & 39,07 & 37,96 & 9,90 \\
\hline$\sigma_{\mathrm{e}}^{2}$ & 91,65 & 91,27 & 91,98 & 63,78 \\
\hline$h^{2}$ & 0,41 & 0,41 & 0,41 & 0,29 \\
\hline $\mathrm{CV}(\%)$ & 8,30 & 8,28 & 8,32 & 6,78 \\
\hline $\mathrm{F}$ & - & $0,69^{\text {ns }}$ & $0,12^{\mathrm{ns}}$ & $293,91 * *$ \\
\hline \multirow[t]{2}{*}{$\mathrm{b}$} & - & $-0,06 * *$ & $0,01 * *$ & $0,44 *$ \\
\hline & & \multicolumn{3}{|c|}{ Covariável "ponta" } \\
\hline$\sigma_{\mathrm{G}}^{2}$ & 91,87 & 91,64 & 91,42 & 20,99 \\
\hline$\sigma_{\mathrm{GxL}}^{2}$ & 38,14 & 38,22 & 37,62 & 2,01 \\
\hline$\sigma_{\mathrm{e}}^{2}$ & 91,65 & 91,81 & 92,13 & 55,12 \\
\hline$h^{2}$ & 0,41 & 0,41 & 0,41 & 0,27 \\
\hline CV $(\%)$ & 8,30 & 8,31 & 8,32 & 6,31 \\
\hline $\mathrm{F}$ & - & $0,36^{\text {ns }}$ & $0,30^{\mathrm{ns}}$ & $512,40 * *$ \\
\hline \multirow[t]{2}{*}{$\mathrm{b}$} & - & $0,0389 *$ & $-0,0269 * *$ & $0,5348^{*}$ \\
\hline & & \multicolumn{3}{|c|}{ Covariável "quatro vizinhos" } \\
\hline$\sigma_{\mathrm{G}}^{2}$ & 91,87 & 91,70 & 92,28 & 8,32 \\
\hline$\sigma_{\mathrm{GxL}}^{2}$ & 38,14 & 37,95 & 38,44 & 1,64 \\
\hline$\sigma_{\mathrm{e}}^{2}$ & 91,65 & 92,00 & 91,70 & 33,07 \\
\hline$h^{2}$ & 0,41 & 0,41 & 0,41 & 0,19 \\
\hline $\mathrm{CV}(\%)$ & 8,30 & 8,32 & 8,31 & 4,94 \\
\hline $\mathrm{F}$ & - & $0,12^{\text {ns }}$ & $0,22^{\mathrm{ns}}$ & $1.164,69 * *$ \\
\hline$\underline{b}$ & - & $0,03 *$ & $0,04 * *$ & $0,74^{\mathrm{ns}}$ \\
\hline
\end{tabular}

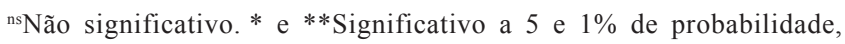
respectivamente. 
por essa metodologia, com uso de qualquer uma das covariáveis também foram menores, em comparação aos outros métodos. Nos dois cortes, a covariável "quatro vizinhos" proporcionou as maiores reduções. Essas reduções podem ter ocorrido em consequência de diferenças existentes entre médias da covariável, entre os genótipos associados, e não em razão de um controle eficiente da variação ambiental. Com esse método, as variâncias genotípica e da interação genótipo $\mathrm{x}$ locais também foram drasticamente reduzidas, em comparação às análises realizadas por Papadakis ou médias móveis 1 (Tabela 1 e 2).

Tabela 2. Estimativas das variâncias genotípicas $\left(\sigma_{\mathrm{G}}^{2}\right)$, de interação genótipos x locais $\left(\sigma_{\text {GxL }}^{2}\right)$ e ambiental $\left(\sigma_{\mathrm{e}}^{2}\right)$, da herdabilidade $\left(\mathrm{h}^{2}\right)$ e do coeficiente de variação $(\mathrm{CV})$, sem e com o uso de covariáveis de competição pelos métodos de Papadakis, médias móveis 1 e médias móveis 2, e valor do teste $\mathrm{F}$ para cada coeficiente de regressão (b), entre as respostas das parcelas e os valores das covariáveis, para o atributo produtividade ( $\mathrm{TCH}, \mathrm{Mg} \mathrm{ha}^{-1}$ ) no terceiro corte de 26 genótipos de cana-de-açúcar, em sete locais.

\begin{tabular}{|c|c|c|c|c|}
\hline \multirow[t]{2}{*}{ Parâmetros } & \multirow{2}{*}{$\begin{array}{c}\text { Análise sem } \\
\text { covariável }\end{array}$} & \multicolumn{3}{|c|}{ Métodos de vizinhança } \\
\hline & & Papadakis & Médias móveis 1 & Médias móveis 2 \\
\hline & & \multicolumn{3}{|c|}{ Covariável "lateral" } \\
\hline$\sigma_{\mathrm{G}}^{2}$ & 80,61 & 80,54 & 77,83 & 19,22 \\
\hline$\sigma_{\mathrm{GxL}}^{2}$ & 36,47 & 36,36 & 35,15 & 2,93 \\
\hline$\sigma_{\mathrm{e}}^{2}$ & 66,16 & 66,38 & 65,71 & 43,20 \\
\hline$h^{2}$ & 0,44 & 0,44 & 0,44 & 0,29 \\
\hline $\mathrm{CV}(\%)$ & 10,31 & 10,33 & 10,27 & 8,00 \\
\hline $\mathrm{F}$ & - & $0,15^{\mathrm{ns}}$ & $6,39 * *$ & $474,84 * *$ \\
\hline \multirow[t]{2}{*}{$\mathrm{b}$} & - & $-0,03^{\text {ns }}$ & $-0,10^{*}$ & $0,47 * *$ \\
\hline & & \multicolumn{3}{|c|}{ Covariável "ponta" } \\
\hline$\sigma_{\mathrm{G}}^{2}$ & 80,61 & 82,17 & 81,19 & 14,55 \\
\hline$\sigma_{\mathrm{GxL}}^{2}$ & 36,47 & 36,47 & 36,84 & 1,52 \\
\hline$\sigma_{\mathrm{e}}^{2}$ & 66,16 & 65,03 & 66,16 & 45,06 \\
\hline$h^{2}$ & 0,44 & 0,45 & 0,44 & 0,24 \\
\hline CV (\%) & 10,31 & 10,22 & 10,29 & 8,27 \\
\hline $\mathrm{F}$ & - & $5,91 * *$ & $0,12^{\mathrm{ns}}$ & $501,79 * *$ \\
\hline \multirow[t]{2}{*}{$\mathrm{b}$} & - & $0,22^{\mathrm{ns}}$ & $0,02 * *$ & $0,53^{\text {ns }}$ \\
\hline & & \multicolumn{3}{|c|}{ Covariável "quatro vizinhos" } \\
\hline$\sigma_{\mathrm{G}}^{2}$ & 80,61 & 81,80 & 78,31 & 6,53 \\
\hline$\sigma_{\mathrm{GxL}}^{2}$ & 36,47 & 36,73 & 34,81 & 0,00 \\
\hline$\sigma_{\mathrm{e}}^{2}$ & 66,16 & 65,67 & 66,75 & 28,93 \\
\hline$h^{2}$ & 0,44 & 0,44 & 0,44 & 0,18 \\
\hline CV $(\%)$ & 10,31 & 10,27 & 10,33 & 6,70 \\
\hline $\mathrm{F}$ & - & $2,44^{\mathrm{ns}}$ & $2,11^{\mathrm{ns}}$ & $1.132,89 * *$ \\
\hline $\mathrm{b}$ & - & $0,20^{\mathrm{ns}}$ & $-0,10 * *$ & $0,68 * *$ \\
\hline
\end{tabular}

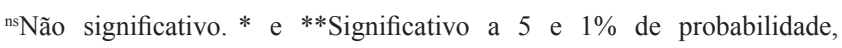
respectivamente.
A herdabilidade também foi intensamente reduzida no método médias móveis 2 , e ficou em torno de $30 \%$ da herdabilidade estimada pelos outros métodos, quando se incluiu a covariável "quatro vizinhos".

Em um estudo com dados simulados, Vivaldi (1990) constatou que, na ausência de tendência linear e na presença de tendências lineares fraca e cíclica, o método médias móveis 2 subestimou o erro experimental em níveis não toleráveis, tendo ficado em 7,53\% da variância do erro estimada pelo método usual. Em relação a essas alterações nas variâncias ambiental e genotípica, Costa et al. (2005) comentaram sobre a possível ocorrência de “overfitting". Provavelmente, ocorre um sobreajuste que faz com que os genótipos sejam parametrizados mais de uma vez, ou seja, na própria parcela do genótipo e na formação da covariável associada.

A covariável "lateral", avaliada pelo método médias móveis 1, e a covariável "ponta", avaliada pelo Papadakis, apresentaram efeitos significativos pelo teste F, no terceiro corte (Tabela 2). Esse resultado indicou que esses métodos identificaram a existência de pelo menos um genótipo que teve sua produtividade influenciada por seus vizinhos. $O$ fato de nenhuma covariável ter apresentado efeito significativo no primeiro corte, para esses métodos, indica possivelmente que, para que a capacidade competitiva dos genótipos seja instalada, é necessário algum tempo para crescimento das raízes no solo. Segundo Donald (1963), uma planta não compete com outra enquanto o conteúdo de água, nutrientes, luz, temperatura, oxigênio e dióxido de carbono estiverem acima das necessidades de ambas. Mas, quando um desses fatores diminui abaixo da demanda necessária das plantas, iniciase a competição. O fato de a cultura passar anos no campo pode proporcionar, no decorrer dos cortes, aumento da competição entre as raízes, na busca por água e nutrientes do solo. Uma possível explicação é que o sistema radicular continua crescendo ao longo dos anos (Vasconcelos \& Casagrande, 2008), e a competição se instala quando o sistema radicular começa a se entrelaçar.

Pelo método médias móveis 2, todas as covariáveis apresentaram efeito significativo a $1 \%$ de probabilidade, e apresentaram valores de $\mathrm{F}$ de elevada magnitude, em comparação aos outros métodos (Tabelas 1 e 2 ). Isso evidencia a alta 
influência dessas covariáveis detectada por este método, embora com as distorções comentadas anteriormente.

É importante ressaltar que, quando o coeficiente de regressão (b) entre as respostas das parcelas e os valores da covariável apresenta sinal positivo $(+)$, ele provavelmente reflete os efeitos de similaridade entre as parcelas adjacentes. No entanto, o coeficiente $b$ negativo indica que alguma parcela pode estar sofrendo prejuízos, enquanto a parcela vizinha se sobressai, ou vice-versa, em razão da competição.

As covariáveis "lateral" (Papadakis) e "ponta" (médias móveis 1), no primeiro corte (Tabela 1), apresentaram valor negativo, porém de baixa magnitude. No terceiro corte (Tabela 2), a covariável "lateral" avaliada tanto pelo método Papadakis quanto por médias móveis 1 apresentou sinal negativo, o que evidencia a existência de uma provável competição entre os genótipos, e pode ser justificado pela faixa de contato ou de competição de $8 \mathrm{~m}$ existente entre os genótipos de sulcos adjacentes. Os vizinhos de ponta, por manterem menor contato, provavelmente exerceram menor influência. A covariável "quatro vizinhos", no geral, apresentou resultados intermediários, o que parece ser uma compensação das outras duas covariáveis, talvez por seu cálculo incluir o das outras. Cargnelutti Filho et al. (2003) concluíram, após estudar cinco tipos de covariáveis de competição em milho pelo método de Papadakis, que a covariável da parcela de referência e as quatro laterais mais próximas foram as mais eficientes na redução da variância ambiental. É importante ressaltar que parcelas constituídas por cinco sulcos também podem amenizar uma possível competição prejudicial entre vizinhos, uma vez que os três sulcos centrais permanecem apenas em competição com o próprio genótipo.

As análises que associaram covariáveis apresentaram tendências similares às que foram obtidas quando cada covariável foi considerada separadamente (Tabela 3 ). A reunião de covariáveis no mesmo modelo pode sofrer possíveis efeitos de colinearidade, de forma que os efeitos da competição entre elas podem ficar mascarados; ou seja, o efeito de uma covariável, aparentemente, fica eliminado pela outra que lhe é correlacionada e vice-versa, mas, o efeito global continua sendo expresso.
As correlações de Spearman (r), obtidas entre os ordenamentos da análise clássica e das análises com covariáveis no primeiro e terceiro cortes, estão apresentados na Tabela 4. Nos dois cortes, as altas correlações indicaram que praticamente não houve alterações no ordenamento dos genótipos, nas análises pelo método de Papadakis e médias móveis 1, com qualquer uma das covariáveis incluídas. O método médias móveis 2 alterou completamente o ordenamento dos genótipos, principalmente no terceiro corte. A validação dos ordenamentos dos genótipos realizados pelos diferentes métodos, quanto à superioridade produtiva, não foram

Tabela 3. Estimativas das variâncias genotípicas $\left(\sigma_{\mathrm{G}}^{2}\right)$ de interação genótipos x locais $\left(\sigma_{\mathrm{GxL}}^{2}\right)$ e ambiental $\left(\sigma_{\mathrm{e}}^{2}\right)$ da herdabilidade $\left(\mathrm{h}^{2}\right)$ e do coeficiente de variação $(\mathrm{CV})$, com o uso das três covariáveis de competição (lateral, ponta e quatro vizinhos) associadas pelos métodos de Papadakis, médias móveis 1 e médias móveis 2 , e valor do teste $F$ para cada coeficiente de regressão (b), entre as respostas das parcelas e os valores das covariáveis, para o atributo produtividade ( $\mathrm{TCH}, \mathrm{Mg} \mathrm{ha}^{-1}$ ) no primeiro e terceiro cortes, de 26 genótipos de cana-de-açúcar, em 7 locais.

\begin{tabular}{|c|c|c|c|}
\hline \multirow[t]{2}{*}{ Parâmetro } & \multicolumn{3}{|c|}{ Métodos de vizinhança } \\
\hline & Papadakis & Médias móveis 1 & Médias móveis 2 \\
\hline$\sigma_{\mathrm{G}}^{2}$ & 92,44 & $\begin{array}{c}\text { Primeiro corte } \\
89,23\end{array}$ & 8,08 \\
\hline$\sigma_{\mathrm{GxL}}^{2}$ & 39,04 & 38,88 & 1,56 \\
\hline$\sigma_{\mathrm{e}}^{2}$ & 90,94 & 90,73 & 32,99 \\
\hline$h^{2}$ & 0,42 & 0,41 & 0,19 \\
\hline $\mathrm{CV}(\%)$ & 8,27 & 8,25 & 4,94 \\
\hline F (lateral) & $3,48^{\text {ns }}$ & $4,35^{*}$ & $2,82^{\mathrm{ns}}$ \\
\hline b (lateral) & $-0,06 * *$ & $-0,26^{*}$ & $0,11 * *$ \\
\hline $\mathrm{F}$ (ponta) & $2,36^{\mathrm{ns}}$ & $5,37^{*}$ & $4,03 *$ \\
\hline $\mathrm{b}$ (ponta) & $0,04^{*}$ & $-0,36^{*}$ & $0,17 * *$ \\
\hline $\mathrm{F}$ (quatro vizinhos) & $2,89^{\mathrm{ns}}$ & $5,28 *$ & $7,96 * *$ \\
\hline $\mathrm{b}$ (quatro vizinhos) & $0,03^{*}$ & $0,64^{*}$ & $0,44 * *$ \\
\hline$\sigma_{\mathrm{G}}^{2}$ & 82,03 & $\begin{array}{c}\text { Terceiro corte } \\
77,81\end{array}$ & 6,16 \\
\hline$\sigma_{\mathrm{GxL}}^{2}$ & 36,42 & 35,28 & 0,00 \\
\hline$\sigma_{\mathrm{e}}^{2}$ & 65,47 & 66,06 & 28,79 \\
\hline$h^{2}$ & 0,45 & 0,43 & 0,18 \\
\hline $\mathrm{CV}(\%)$ & 10,26 & 10,27 & 6,69 \\
\hline F (lateral) & $0,00^{\mathrm{ns}}$ & $1,37^{\mathrm{ns}}$ & $4,84^{*}$ \\
\hline $\mathrm{b}$ (lateral) & $0,00^{\text {ns }}$ & $-0,15^{\text {ns }}$ & $0,16^{\mathrm{ns}}$ \\
\hline $\mathrm{F}$ (ponta) & $0,53^{\mathrm{ns}}$ & $0,09^{\mathrm{ns}}$ & $3,39^{\text {ns }}$ \\
\hline $\mathrm{b}$ (ponta) & $0,25^{\text {ns }}$ & $-0,05^{\text {ns }}$ & $0,17^{\text {ns }}$ \\
\hline $\mathrm{F}$ (quatro vizinhos) & $0,01^{\mathrm{ns}}$ & $0,13^{\text {ns }}$ & $4,64 *$ \\
\hline $\mathrm{b}$ (quatro vizinhos) & $-0,06^{\mathrm{ns}}$ & $0,10^{\text {ns }}$ & $0,35^{\mathrm{ns}}$ \\
\hline
\end{tabular}

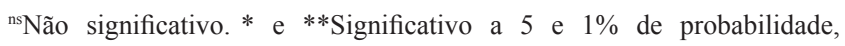
respectivamente. 
validados neste estudo. No entanto, a opinião de melhoristas envolvidos no programa de melhoramento do IAC foi que o ordenamento realizado pelos dois primeiros métodos pareceu mais coerente. Assim, a adoção de uma metodologia de análise de vizinhança, a fim de aumentar a precisão experimental, necessita

Tabela 4. Ordenamento em termos de potencial produtivo de 22 genótipos de cana-de-açúcar ${ }^{(1)}$, avaliados no primeiro e no terceiro cortes, segundo os métodos de vizinhança de Papadakis, médias móveis 1 e médias móveis 2, quando usadas as covariáveis de competição lateral (L), ponta (P) e quatro vizinhos (4 V); e correlações de Spearman (r) entre o ordenamento da análise sem uso de covariável e as análises de vizinhança.

\begin{tabular}{|c|c|c|c|c|c|c|c|c|c|c|}
\hline \multirow[t]{2}{*}{ Ordem } & \multirow[t]{2}{*}{ Sem covariável } & \multicolumn{3}{|c|}{ Papadakis } & \multicolumn{3}{|c|}{ Médias móveis 1} & \multicolumn{3}{|c|}{ Médias móveis 2} \\
\hline & & $\mathrm{L}$ & $\mathrm{P}$ & $4 \mathrm{~V}$ & $\mathrm{~L}$ & $\mathrm{P}$ & $4 \mathrm{~V}$ & $\mathrm{~L}$ & $\mathrm{P}$ & $4 \mathrm{~V}$ \\
\hline & & \multicolumn{9}{|c|}{ Primeiro corte } \\
\hline $1^{\circ}$ & 7 & 7 & 7 & 7 & 7 & 7 & 7 & 7 & 2 & 1 \\
\hline $2^{\circ}$ & 2 & 2 & 2 & 2 & 2 & 2 & 2 & 13 & 4 & 12 \\
\hline $3^{\circ}$ & 13 & 13 & 13 & 13 & 13 & 13 & 13 & 1 & 1 & 16 \\
\hline $4^{\circ}$ & 1 & 1 & 1 & 1 & 1 & 1 & 1 & 2 & 12 & 13 \\
\hline $5^{\circ}$ & 4 & 4 & 4 & 4 & 4 & 4 & 4 & 15 & 16 & 2 \\
\hline $6^{\circ}$ & 12 & 12 & 12 & 12 & 12 & 12 & 12 & 5 & 22 & 4 \\
\hline $7^{\circ}$ & 18 & 18 & 18 & 18 & 18 & 18 & 18 & 14 & 13 & 15 \\
\hline $8^{\circ}$ & 5 & 5 & 5 & 5 & 5 & 16 & 5 & 12 & 7 & 22 \\
\hline $9^{\circ}$ & 16 & 16 & 16 & 16 & 16 & 5 & 14 & 16 & 15 & 20 \\
\hline $10^{\circ}$ & 14 & 14 & 14 & 14 & 14 & 14 & 16 & 4 & 5 & 5 \\
\hline $11^{\circ}$ & 19 & 22 & 22 & 22 & 19 & 22 & 19 & 20 & 20 & 7 \\
\hline $12^{\circ}$ & 22 & 19 & 19 & 19 & 22 & 19 & 22 & 22 & 19 & 14 \\
\hline $13^{\circ}$ & 15 & 15 & 15 & 15 & 15 & 15 & 15 & 19 & 18 & 19 \\
\hline $14^{\circ}$ & 21 & 21 & 21 & 21 & 21 & 21 & 21 & 21 & 21 & 21 \\
\hline $15^{\circ}$ & 20 & 20 & 20 & 20 & 20 & 20 & 20 & 6 & 14 & 6 \\
\hline $16^{\circ}$ & 9 & 9 & 9 & 9 & 9 & 9 & 9 & 18 & 9 & 11 \\
\hline $17^{\circ}$ & 6 & 6 & 6 & 6 & 6 & 6 & 6 & 11 & 11 & 18 \\
\hline $18^{\circ}$ & 11 & 11 & 11 & 11 & 11 & 11 & 11 & 9 & 6 & 17 \\
\hline $19^{\circ}$ & 17 & 17 & 17 & 17 & 17 & 17 & 17 & 17 & 10 & 10 \\
\hline $20^{\circ}$ & 10 & 10 & 10 & 10 & 10 & 10 & 10 & 10 & 17 & 9 \\
\hline $21^{\circ}$ & 8 & 8 & 8 & 8 & 8 & 8 & 8 & 8 & 3 & 3 \\
\hline $22^{\circ}$ & 3 & 3 & 3 & 3 & 3 & 3 & 3 & 3 & 8 & 8 \\
\hline \multirow[t]{2}{*}{$r$} & - & $0,98^{* *}$ & $0,98^{* *}$ & $0,98 * *$ & 1,0 & $0,85^{* *}$ & $0,99 * *$ & $0,47 * *$ & $0,49 * *$ & 0,17 \\
\hline & & \multicolumn{9}{|c|}{ Terceiro corte } \\
\hline $1^{\circ}$ & 22 & 22 & 22 & 22 & 22 & 22 & 22 & 13 & 4 & 1 \\
\hline $2^{\circ}$ & 7 & 7 & 7 & 7 & 7 & 7 & 7 & 7 & 1 & 4 \\
\hline $3^{\circ}$ & 4 & 4 & 4 & 4 & 13 & 4 & 4 & 1 & 2 & 13 \\
\hline $4^{\circ}$ & 13 & 13 & 13 & 13 & 1 & 13 & 1 & 22 & 22 & 16 \\
\hline $5^{\circ}$ & 1 & 1 & 2 & 1 & 4 & 1 & 13 & 4 & 13 & 2 \\
\hline $6^{\circ}$ & 2 & 2 & 1 & 2 & 2 & 2 & 2 & 2 & 18 & 20 \\
\hline $7^{\circ}$ & 21 & 21 & 21 & 21 & 21 & 21 & 21 & 21 & 5 & 15 \\
\hline $8^{\circ}$ & 18 & 18 & 18 & 18 & 18 & 18 & 18 & 20 & 21 & 22 \\
\hline $9^{\circ}$ & 5 & 5 & 5 & 5 & 5 & 5 & 5 & 5 & 20 & 21 \\
\hline $10^{\circ}$ & 20 & 20 & 20 & 20 & 20 & 20 & 20 & 18 & 16 & 6 \\
\hline $11^{\circ}$ & 17 & 17 & 17 & 17 & 17 & 17 & 17 & 15 & 19 & 5 \\
\hline $12^{\circ}$ & 9 & 9 & 19 & 9 & 19 & 9 & 19 & 16 & 17 & 12 \\
\hline $13^{\circ}$ & 19 & 19 & 9 & 19 & 9 & 19 & 9 & 6 & 9 & 18 \\
\hline $14^{\circ}$ & 16 & 16 & 16 & 16 & 16 & 16 & 16 & 19 & 7 & 19 \\
\hline $15^{\circ}$ & 12 & 12 & 12 & 12 & 12 & 12 & 12 & 17 & 12 & 7 \\
\hline $16^{\circ}$ & 14 & 14 & 14 & 14 & 14 & 14 & 14 & 8 & 6 & 9 \\
\hline $17^{\circ}$ & 15 & 15 & 6 & 6 & 15 & 15 & 15 & 9 & 15 & 17 \\
\hline $18^{\circ}$ & 6 & 6 & 15 & 15 & 6 & 6 & 6 & 14 & 11 & 11 \\
\hline $19^{\circ}$ & 11 & 11 & 8 & 11 & 8 & 11 & 11 & 12 & 14 & 14 \\
\hline $20^{\circ}$ & 8 & 8 & 11 & 8 & 11 & 8 & 8 & 11 & 3 & 8 \\
\hline $21^{\circ}$ & 10 & 10 & 10 & 10 & 10 & 10 & 10 & 10 & 8 & 10 \\
\hline $22^{\circ}$ & 3 & 3 & 3 & 3 & 3 & 3 & 3 & 3 & 10 & 3 \\
\hline $\mathrm{r}$ & - & 1,00 & $0,78^{* *}$ & $0,90^{* *}$ & $0,74 * *$ & 1,00 & $0,72^{* *}$ & $0,66^{* *}$ & 0,00 & 0,08 \\
\hline
\end{tabular}

${ }^{(1)}$ Quinze clones IAC (numeradas de 1 a 15) e sete cultivares padrões: três fixas (numeradas de 16 a 18) e quatro móveis (numeradas de 19 a 22), que variaram de acordo com o local do experimento. **Significativo a $1 \%$ de probabilidade pelo teste $t$. 
ser previamente avaliada, pois pode comprometer, eventualmente, o sucesso da seleção dos genótipos nos programas de melhoramento.

\section{Conclusões}

1. Os métodos de Papadakis e médias móveis 1 não alteram a precisão experimental, bem como o ordenamento dos genótipos, em comparação à análise clássica.

2. O método médias móveis 2 reduz significativamente a variância residual, provoca fortes reduções nas estimativas da variância ambiental e genotípica, e pode causar distorções na avaliação da herdabilidade e no ordenamento dos genótipos em termos do potencial produtivo.

3. A covariável "lateral", por considerar um espaço maior de competição entre os sulcos adjacentes das parcelas é, possivelmente, a mais influente nos efeitos da competição, especialmente no terceiro corte, mas o efeito em parcelas com cinco sulcos é pequeno.

\section{Agradecimentos}

Ao Centro de Cana do Instituto Agronômico, pelo fornecimento dos dados e à CAPES pelo apoio financeiro.

\section{Referências}

AMARO, R.; COBO, M.; QUIROZ, A. Comparación de métodos de ajuste en el análisis espacial de experimentos de campo. Agronomía Tropical, v.47, p.359-374, 1997.

CARGNELUTTI FILHO, A.; STORCK, L.; LUCIO, A.D. Ajustes de quadrado médio do erro em ensaios de competição de cultivares de milho pelo método de Papadakis. Pesquisa Agropecuária Brasileira, v.38, p.467-473, 2003.

COSTA, J.R.; BUENO FILHO, J.S. de S.; RAMALHO, M.A.P. Análise espacial e de vizinhança no melhoramento genético de plantas. Pesquisa Agropecuária Brasileira, v.40, p.1073-1079, 2005.

DONALD, C.M. Competition among crop and pasture plants. Advances in Agronomy, v.15, p.1-118, 1963.

HENDERSON, C.R. Best linear unbiased estimation and prediction under a selection model. Biometrics, v.31, p.423-447, 1975.

PATTERSON, H.D.; THOMPSON, R. Recovery of inter-block information when block sizes are unequal. Biometrika, v.58, p.545-554, 1971.

RAMALHO, M.A.P.; FERREIRA, D.F.; OLIVEIRA, A.C. Experimentação em genética e melhoramento de plantas. Lavras: UFLA, 2005. 300p.

RESENDE, M.D.V. de. Análise estatística de modelos mistos via REML/BLUP na experimentação em melhoramento de plantas perenes. Colombo: Embrapa Florestas, 2000. 101p. (Embrapa Florestas. Documentos, 47).

SAS INSTITUTE. SAS user's guide. Version 9.1. Cary: SAS Institute, 2002.

SOUZA, E.A. de; GERALDI, I.O.; RAMALHO, M.A.P. Alternativas experimentais na avaliação de famílias em programas de melhoramento genético do feijoeiro. Pesquisa Agropecuária Brasileira, v.35, p.1765-1771, 2000.

STORCK, L.; CARGNELUTTI FILHO, A.; LUCIO, A.D.; LOPES, S.J. Método de Papadakis e número de repetições em experimentos de soja. Ciência Rural, v.39, p.977-982, 2009.

STORCK, L.; GARCIA, D.C.; LOPES, S.J.; ESTEFANEL, V. Experimentação vegetal. 2.ed. Santa Maria: Universidade Federal de Santa Maria, 2006. 198p.

STORCK, L.; STECKLING, C.; ROVERSI, T.; LOPES, S.J.Utilização do método de Papadakis na melhoria da qualidade experimental de ensaios com soja. Pesquisa Agropecuária Brasileira, v.43, p.581-587, 2008.

VASCONCELOS, A.C.M. de; CASAGRANDE, A.A. Fisiologia do sistemaradicular.In:DINARDO-MIRANDA,L.L.; VASCONCELOS, A.C.M. de; LANDELL, M.G. de A. (Ed.). Cana-de-açúcar. Campinas: Instituto Agronômico, 2008. 882p.

VIVALDI, L.J. Comparação entre métodos de análise espacial de experimentos de campo. Pesquisa Agropecuária Brasileira, v.25, p.77-84, 1990.

$\overline{\text { Recebido em } 27 \text { de abril de } 2009 \text { e aprovado em } 26 \text { de setembro de } 2009}$ 